International Mathematical Forum, 2, 2007, no. 33, 1617 - 1623

\title{
Mathematical Approach to Vagueness
}

\author{
Angel Garrido \\ Departamento de Matematicas Fundamentales \\ Facultad de Ciencias de la UNED \\ Senda del Rey, 9, 28040-Madrid, Spain \\ algbmv@telefonica.net
}

\begin{abstract}
From 1920, when Lukasiewicz established the 3-valued logic, many other versions have been made, with the purpose of improving or generalizing the precedent system. Fuzzy Logic departs from the multi-valued logic point of view, with real truth values in the closed unit interval.

Our paper deals, basically, with analogies and differences between fuzzy and rough characters. Therefore, it belongs to a relatively new field: Fuzzy/Rough Mathematical Analysis.

The Fuzzy Theory was initiated by Lofti A. Zadeh, in 1965, and the Rough Theory by Zdzislaw Pawlak, in 1981-82. As general purpose, to obtain a way to model flexible restrictions constructed from vague pieces of information. From then, great advances have followed, not only in theoretical aspects, but in applications.

We attempt to give a clear vision of the foundations and applications of such very related, but essentially different, theories.
\end{abstract}

Mathematics Subject Classification: 03E72, 94D05, 93C41, 68727

Keywords: Fuzzy Set Theory, Fuzzy Sets and Logic, Problems with incomplete information, Logics in A. I.

\section{Introduction}

From 1920, when Lukasiewicz introduced his 3-valued logic, many other versions have been developed, aiming to improve or generalize the precedent system. Among them, the best known is the logic system of Kleene. It consists of a special case in the logic system of Lukasiewicz. And in line with this, the Fuzzy Logic departs from the multi-valued logic point of view, with real truth values into the closed unit interval. 
So, in a previous step (Classical Logic) we have two truth values: 0 and 1 . In the subsequent step (Lukasiewicz), we dispose of three truth values: $0,1 / 2$ and 1 .

In an intermediate step, when it is the n-valued logic, we will have $n$ possible truth values: $0,1 / \mathrm{n}-1,2 / \mathrm{n}-1, \ldots .,(\mathrm{n}-1) / \mathrm{n}-1=1, n$ rational numbers in the closed unit interval.

And the final step will be all the closed unit interval as range of truth values. Therefore, the Fuzzy system is an infinite-valued logic.

\section{On Fuzziness}

It is possible to introduce new generalized versions of Classical Logic. That is the case with the Modus Ponens Generalized, or the Modus Tollens Generalized, or the Hypothetic Syllogism.

To each Fuzzy Predicate, we will associate a Fuzzy Set: the one defined by such property, that is, composed by the elements of the Universe such that totally or partially verify that condition.

So, we can prove that the class of fuzzy sets with the usual operations of union, intersection and path to the complementary does not constitute a Boolean Algebra, because neither the Contradiction Law nor the Third Excluded Principle work in it. Geometrical and algebraic proofs are easy, by a counterexample: it suffices to take an element with membership degree that belongs to the open unit interval: $0<\mu(x)<1$.

Summing up the aforementioned concepts, we will formulate this result:

Theorem 2.1 There exists an isomorphism between the infinite-valued logic and the Fuzzy Set Theory.

Suppose a fuzzy or vague predicate, $P$, in the infinite-valued logic. As you know, the truth value set in this case will be: $T_{\infty}=[0,1]$. Then, $P(x)$ or equivalently $x \in P$ will be identified as its membership degree, that is, the intensity of $x$ in the accomplishment of the corresponding property, described by $P$.

And reciprocally, if we depart from the number $\mu_{P}(x)$ in the closed unit interval, named membership degree of $x$ to $P$, then it will be interpreted as the truth value of $x \in P$, in our infinite-valued logic.

This implies, obviously, that in the same way that Fuzzy Logic is an extension of Classical Logic, Fuzzy Set Theory is a generalization of Classical Set Theory.

Furthermore, we would introduce all the linguistic modifiers and with them, the structure and derived problems of natural language. So appears the Approximate Reasoning. 


\section{On Roughness}

The concept of Rough Set was introduced by the polish mathematician Zdzislaw Pawlak in 1982. Taking objects, attributes or decision values, we will create rules for them: upper and lower approximations and boundary approximation. Each object is classified in one of these regions. For each rough set, $A \subset U$, we dispose of:

- Lower Approximation of A: collection of objects which can be classified with full certainty as members of A.

- Upper Approximation of A: collection of objects which may possibly be classified as members of $\mathrm{A}$.

Obviously, this class is wider than the aforementioned, containing "between both" the Rough set.

The process will be articulated in three steps:

First Step: We depart from a relational database, composed by a table of objects with their attributes and the corresponding values.

Second Step: We put together such objects according to their attributes and the corresponding values of such attributes on each class. So, we obtain a classification. In mathematical terms, a partition in equivalence classes.

Third Step: It is possible to form the Indiscernibility Matrix, showing the equivalence classes and the attribute values on each class.

The relation between Knowledge Theory and Rough Sets will be in different forms. For instance, in Approximate Reasoning, Pattern Recognition, Machine Intelligence and so on.

But not every attribute is needed to characterize each class. We will define the Relative Reduction concept. It contains enough information to distinguish between the different classes.

Rough Set Theory is a model of Approximate Reasoning. According to it we will interpret knowledge as a way to classify objects.

We say that an object, or category, is $R$-rough, if it is not R-exact.

For each R-rough set, $Y \subset U$, we define two associate R-exact sets:

- the $R$-lower approximation of $\mathrm{Y}$ :

$$
\underline{R} Y=\left\{x \in U:[x]_{R} \subseteq Y\right\}
$$

-the $R$-upper approximation of $\mathrm{Y}$ :

$$
\bar{R} Y=\left\{x \in U:[x]_{R} \cap Y \neq \emptyset\right\}
$$


then, we can represent the Rough set, $Y$, through the pair:

$$
(\underline{R} Y, \bar{R} Y)
$$

Observe that:

Pr. 1:

$$
\underline{R} Y \subset Y \subset \bar{R} Y, \forall Y \subseteq U
$$

As model of proof, we show this:

Let $x$ be an element of the universe, $U$, such that:

$$
x \in \underline{R} Y
$$

Then:

$$
[x]_{R} \subseteq Y
$$

Therefore:

$$
[x]_{R} \cap Y \neq \emptyset
$$

We omit the subsequent detailed proofs, because they require very similar reasoning.

Pr. 2:

$$
Y \text { is } R-\text { exact } \Leftrightarrow \underline{R} Y=\bar{R} Y
$$

Given a Knowledge Base: $K \equiv(U, R)$, we will take the collection of classes:

$$
E_{K}=\{R-\text { exact sets on } U\}
$$

that is closed with respect to usual set operations: $\cup, \cap$ andc. It verifies the known properties of a Boolean Algebra. More concretely, we can say a Field of Sets.

But it is not the case when we deal with $R$-rough sets. Because, for instance, the union of two R-rough sets can be a R-exact set. For this reason, we represent the Rough set as a pair $(A, B)$, where $A$ and $B$ are two exact sets such that: $A \cap B=\emptyset$.

Many algebraic structures can be applied to the representation of Rough Sets. For instance: the Nelson Algebra, the Post Algebra, the Lukasiewicz Algebra and so on. 
The above approximation operators give this sequence of enchained results:

- Lower-approximation:

Pr. 3:

$$
\underline{R}(A \cap B)=\underline{R} A \cap \underline{R} B
$$

Pr. 4:

$$
\underline{R} A \subseteq A
$$

Pr. 5:

$$
\underline{R}(\underline{R} A)=\underline{R} A
$$

Pr. 6:

$$
\underline{R} \emptyset=\emptyset
$$

- Upper-approximation:

Pr. 7:

$$
\bar{R}(A \cup B)=\bar{R} A \cup \bar{R} B
$$

Pr. 8:

$$
A \subseteq \bar{R} A
$$

Pr. 9:

$$
\bar{R}(\bar{R} A)=\bar{R} A
$$

Pr. 10:

$$
\bar{R} U=U
$$

There exist the corresponding topological properties on interior and closure of sets.

The Rough Set Theory is related (as we know) to the 3-valued logic of Lukasiewicz and Moisil, in the form of Lindebaum-Tarski Algebra, with three truth values: $0,1 / 2$ and 1 . Because when we interpret a Rough set as a pair of exact sets: $(A, B)$, we automatically induce three objects:

$A$, representing the logical value of truth

$U \backslash B$, containing the logical value of falsity

and

$(U \backslash B) \backslash A$, representing the third case: of uncertainty

It can also be related with the Modal Logic (ML), with its concepts and associated operators of: Necessity $(\square)$ and Possibility $(\diamond)$.

The immanent character of these ideas in ML is shown in this form: Let $\mathrm{Y}$ be a Rough Set and B a collection of attributes. When we say: 


$$
x \in \underline{B} Y
$$

it means that $x$ belongs necessarily to $Y$.

Whereas when we say:

$$
x \in \bar{B} Y
$$

we are saying that $x$ belongs possibly to $Y$.

Let $\mathrm{U}$ be a universe, $\mathrm{X}$ a set of objects in $\mathrm{U}$ and $\mathrm{B}$ a set of attributes. Then, we proceed to introduce the Rough Membership Function:

$$
\mu_{X}^{B}:(X, B) \rightarrow[0,1]
$$

by the assignation, for each $x$, to its membership degree:

$$
\mu_{X}^{B}(x) \equiv \frac{\operatorname{card}\left([x]_{B} \cap X\right)}{\operatorname{card}\left([x]_{B}\right)}
$$

Obviously:

Pr. 11:

$$
X \text { is } R-\text { exact } \Leftrightarrow \mu_{X}^{B} \equiv \chi_{X}
$$

that is, it coincides with its characteristic function.

Therefore, the coincidence of this Theory with the Classical Theory of Sets occurs when we only work with R-exact sets.

Nowadays the principal trends in Rough Set research are:

- To reach the Robustness of Decision Rules. Therefore, we attempt to obtain the stability with respect to small changes in the information set of objects.

- To reach noise-resistance. That is, the preservation of the way of classification when the noise is present.

For such purposes, we need dispose of:

- Adequate set of attributes

- Adequate techniques in the search of Proper Patterns and Decomposition Methods.

In subsequent papers, we will analyze the mutual relationship between both theories and the mutual support in the advances through different fields. 


\section{References}

[1] Cattaneo, Abstract approximation spaces for rough theories Rough Sets in Knowledge, Physica-Verlag, Heidelberg, pp. 59-98.

[2] Farinas del Cerro and Prade, Rough Sets, twofold fuzzy sets and modal logic-fuzziness in indiscernibility classes and partial information, in The Mathematics of Fuzzy Systems, Verlag TUV Rheinland, Koln, 1986.

[3] Garrido, Logical Foundations of Fuzzy Theory, International Conference Foundations of Formal Sciences: Infinite Games, FotFs V Univ. of Bonn, 2004.

[4] Mántaras and Valverde, New results in fuzzy clustering based on the concept of indisguishability relation, IEEE Pattern Analysis, pp. 754-757, 1988.

Received: November 8, 2006 